doi: http://dx.doi.org/10.7124/bc.0009D1

\section{B-1. Nucleolar localization of EGFR with different status in lung adenocarcinoma cells}

E. Baidyuk, O. Shuvalov, A. Daks, O. A. Fedorova, M. Vorobev, M. Piacentini, N. Barlev

Institute of Cytology of the Russian Academy of Sciences, 194064 Tikhoretskyave. 4, Saint-Petersburg, Russia

katya_bay@mail.ru

The aim of the work was to assess the localization of EGFR in the cells of lung adenocarcinoma depending on the presence of various mutations in the EGFR gene, characterized by different resistance to inhibitors of tyrosine kinase activity of EGFR. Methods: To investigate the effect of resistance-conferring mutations in EGFR common for non-small cell lung cancer we monitored the intracellular localization of EGFR in H1299 cell line (expresses wild type EGFR). By using CRISPR/Cas9 gene editing system we introduced specific mutations in the 20 and 21 exons of the EGFR gene either as single (H1299/T790M and H1299/L858R, respectively) or double mutations (H1299/ L858R/T790M). Additionally, H1975 cells, also carrying a double mutation (in 20 and 21 exons) and $\mathrm{H} 1650$ cells, carrying a mutation in the 19 exon of the EGFR gene were analyzed. As control, MDA-MB 468 cell line (breast cancer cells) was used, for which a clear membrane localization of EGFR has been established. Primary antibodies (EGFR polyclonal antibody, Santa Cruz, USA) and secondary antibodies
(Alexa fluor 546, Invitrogen, USA) were used in immunocytochemical analysis. Cell images were obtained using a confocal microscope Olympus FV3000 (Olympus Corporation, Germany). Results: EGFR displayed clear cellular membrane localization in control MDA468 cells and was not observed in the nuclei and nucleolus of cells. Wild type EGFR in H1299 cells behaved differently whereby the membrane signal of EGFR was weaker than in MDA468 cells. In addition, EGFR localization in the nucleus and nucleolus of cells was also detected. In H1299 L858R cells, as well as in cells with double mutations (H1299 L858R/ T790M), EGFR was detected both in the nucleus and nucleolus, but the membrane localization of the EGFR receptor was missing. In H1299 T790M cells, EGFR was present in the nucleus, nucleolus and membrane of cells, thus repeating the staining pattern of H1299 WT. Notably, EGFR in H1975 cells also exhibited a nucleolar localization. H1650 cells with mutan tEGFR as well as H1299 cells with L858R mutant EGFR were detected in the nucleus and nucleolus of cells, but not on the cell membrane. It is known that EGFR participates in the regulation of autophagy. We used the well-known inhibitor, Chloroquine, to monitor the distribution of EGFR. We found that treatment of H1299 cells with this drug had differential effect on the localization of mutant EGFR. Conclusions: Thus, EGFR, as a membrane receptor, exhibits aberrant localization in lung cancer cells, which can contribute to the development of resistance to chemotherapy.

Mega-grant 14.W03.31.0029. 